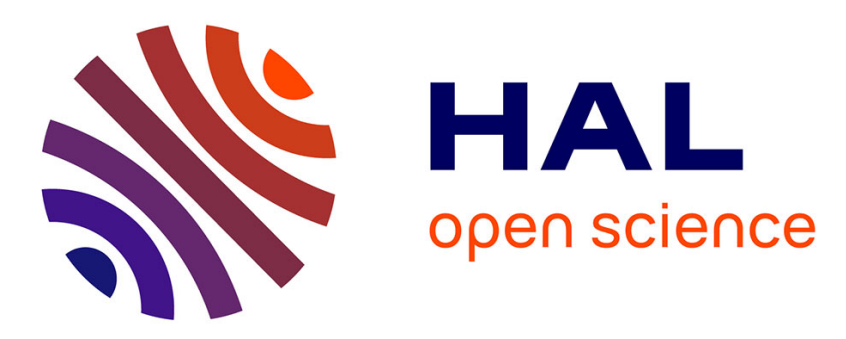

\title{
A shell-model calculation in terms of correlated subsystems
}

J.P. Boisson, B. Silvestre-Brac, R.J. Liotta

\section{To cite this version:}

J.P. Boisson, B. Silvestre-Brac, R.J. Liotta. A shell-model calculation in terms of correlated subsystems. Journal de Physique Lettres, 1979, 40 (13), pp.263-265. 10.1051/jphyslet:019790040013026300 . jpa-00231621

\section{HAL Id: jpa-00231621 https://hal.science/jpa-00231621}

Submitted on 1 Jan 1979

HAL is a multi-disciplinary open access archive for the deposit and dissemination of scientific research documents, whether they are published or not. The documents may come from teaching and research institutions in France or abroad, or from public or private research centers.
L'archive ouverte pluridisciplinaire HAL, est destinée au dépôt et à la diffusion de documents scientifiques de niveau recherche, publiés ou non, émanant des établissements d'enseignement et de recherche français ou étrangers, des laboratoires publics ou privés. 


\title{
A shell-model calculation in terms of correlated subsystems
}

\author{
J. P. Boisson, B. Silvestre-Brac \\ Institut des Sciences Nucléaires, 53, avenue des Martyrs, 38026 Grenoble Cedex, France
}

and R. J. Liotta

Research Institute of Physics, S-10404 Stockholm 50, Sweden

(Reçu le 22 janvier 1979, révisé le 14 mai 1979, accepté le 17 mai 1979)

\begin{abstract}
Résumé. - Nous présentons une méthode pour résoudre les équations du modèle en couches à l'aide d'une base qui contient des sous-systèmes corrélés. Il est montré que la méthode permet d'effectuer des troncatures importantes de la base. Les calculs correspondants sont faciles à mettre en œuvre et peuvent être effectués de façon très rapide.
\end{abstract}

\begin{abstract}
A method for solving the shell-model equations in terms of a basis which includes correlated subsystems is presented. It is shown that the method allows drastic truncations of the basis to be made. The corresponding calculations are easy to perform and can be carried out rapidly.
\end{abstract}

It is now well established that the shell-model approach [1] is a powerful tool to deal with the manybody problem in Nuclear Physics.

However, when the number of valence particles or the number of single-particle orbits is large, the shellmodel method may become difficult to apply and the corresponding results difficult to interpret. In addition, a truncation of large standard shell-model basis may not be a reasonable possibility if collective states (i.e. states with many relevant elementary configurations) are important. To deal with cases like this, models have been introduced which consider correlated pairs of particles as elementary degrees of freedom [2-5]. As a drawback, the basis used by these models may be overcomplete and spurious states may appear [3, 4]. Moreover, in some of these models the calculation of the interaction matrix elements can be a difficult task [2, 4-5] and sometimes the wavefunctions are not properly normalized [3].

In this letter, we present a method which also contains correlated pairs (or, in general, correlated subsystems) in the overcomplete basis but without the other inconveniences mentioned above. We first show the general features of the method and then we give some details for systems with three identical particles outside an inert closed shell core.

Let us consider a system with a given number of valence particles. Taking into account all the correlated two particle states one then obtains a basis consisting of $N$ vectors $\left|\varphi_{i}\right\rangle(i=1,2, \ldots, N)$. These $N$ vectors span the shell model space $\varepsilon_{n}$ and in most cases they are linearly dependent $(N>n$; this set of $N$ vectors is called an overcomplete basis).

Since an important aim of the method is to be able to perform drastic truncations, we further assume that from the original overcomplete basis a number $N^{\prime} \leqslant N$ of vectors are chosen. These $N^{\prime}$ vectors span the shell-model subspace $\varepsilon_{n^{\prime}}$ of dimension $n^{\prime}$ with $n^{\prime} \leqslant n$. Given the overlap matrix between these $N^{\prime}$ vectors

$$
\Delta_{i j}=\left\langle\varphi_{i} \mid \varphi_{j}\right\rangle
$$

one can utilize any of the available method to generate $n^{\prime}$ orthonormalized vectors $\left|m_{p}\right\rangle$ of $\varepsilon_{n^{\prime}}$

$$
\left|m_{p}\right\rangle=\sum_{i=1}^{N^{\prime}} \xi_{p}(i)\left|\varphi_{i}\right\rangle ; \quad p=1,2, \ldots, n^{\prime}
$$

To solve the Schrödinger equation within the space $\varepsilon_{n^{\prime}}$ it is sufficient to calculate the interaction matrix element between the states (2), i.e.

$\left\langle m_{p}|\mathcal{H}| m_{q}\right\rangle=\sum_{i, j=1}^{N^{\prime}} \xi_{p}^{*}(i) \xi_{q}(j)\left\langle\varphi_{i}|\mathcal{H}| \varphi_{j}\right\rangle$.

As mentioned above, the evaluation of the matrix elements in the right-hand side of eq. (3) can be cumbersome [2, 4-5]. However, we notice that the 
effective shell-model Hamiltonian $\mathscr{H}$ acts upon the space $\varepsilon_{n}$ and one can thus write

$$
\begin{aligned}
\mathscr{H}\left|\varphi_{j}\right\rangle & =\sum_{k=1}^{N} A_{j k}\left|\varphi_{k}\right\rangle \\
\left\langle\varphi_{i}|\mathcal{H}| \varphi_{j}\right\rangle & =\sum_{k=1}^{N} A_{j k} \Delta_{i k} .
\end{aligned}
$$

Since the basis is overcomplete the coefficients $A_{j k}$ are not uniquely determined. One may then be able to select values of $A_{j k}$ which allow easy calculation of the matrix elements (5), as seen below.

The diagonalization of the matrix (3) provides the orthonormalized wavefunctions

$$
\left|\psi_{\beta}\right\rangle=\sum_{p=1}^{n^{\prime}}\left\langle m_{p} \mid \psi_{\beta}\right\rangle\left|m_{p}\right\rangle
$$

or

$$
\left|\psi_{\beta}\right\rangle=\sum_{i=1}^{N^{\prime}} X_{\beta}(i)\left|\varphi_{i}\right\rangle
$$

where

$$
X_{\beta}(i)=\sum_{p=1}^{n^{\prime}}\left\langle m_{p} \mid \varphi_{\beta}\right\rangle \xi_{p}(i) .
$$

With the wavefunction (6) one can proceed to calculate the matrix elements of any transition operator.

Let us point out a very important and general feature of the method. In order to find the results of the standard shell model approach it is sufficient to consider a set of $N^{\prime}$ vectors $\left|\varphi_{i}\right\rangle$ which span the shellmodel space $\varepsilon_{n}\left(n^{\prime}=n\right)$. In practice this is obtained with $N^{\prime}$ equal to $n$ plus a few units.

As an illustration, we consider the case of three identical particles outside a closed-shell nucleus. The overcomplete basis $\left|\varphi_{i}\right\rangle$ is in this case given by

$$
\left|\varphi_{m \alpha J ; I M}\right\rangle=\left[C_{m}^{+} P_{\alpha}^{+}(J)\right]_{I M}|0\rangle
$$

where $C_{m}^{+}$is a fermion creation operator and $P_{\alpha}^{+}$is a two-particle TDA creation operator. Making use of both the two-particle and the three-particle TDA equations one finds that the bare interaction matrix elements can be replaced by the corresponding twoparticle energies and wavefunction components $[3,6]$. The resulting three-particle TDA equation is then written in terms of the vector (8). From this equation one obtains a matrix $A$ which has the value

$$
A_{m \alpha J, m^{\prime} \alpha^{\prime} J^{\prime}}=\left(\varepsilon_{m}+\omega_{\alpha}(J)\right) \delta_{m m^{\prime}} \delta_{\alpha \alpha^{\prime}} \delta_{J J^{\prime}}+\sum_{p}\left(\omega_{\alpha^{\prime}}\left(J^{\prime}\right)-\varepsilon_{m}-\varepsilon_{p}\right) M_{p}\left(m^{\prime} \alpha^{\prime} J^{\prime}, m \alpha J\right)
$$

where

$$
\begin{array}{r}
M_{p}\left(m^{\prime} \alpha^{\prime} J^{\prime}, m \alpha J\right)=\left[(2 J+1)\left(2 J^{\prime}+1\right)\right]^{1 / 2}\left\{\begin{array}{rrr}
j_{m} & j_{p} & J^{\prime} \\
j_{m^{\prime}} & I & J
\end{array}\right\}\left\langle 0\left|P_{\alpha^{\prime}}\left(J^{\prime}\right)\left[C_{p}^{+} C_{m}^{+}\right]_{J^{\prime}}\right| 0\right\rangle \times \\
\times\left\langle 0\left|P_{\alpha}(J)\left[C_{p}^{+} C_{m^{\prime}}^{+}\right]_{J}\right| 0\right\rangle^{*} .
\end{array}
$$

Here $\omega_{\alpha}$ is a two-particle correlated energy and $\varepsilon_{m}$ is a single particle energy. An important property of the matrix $A$ given by (9) is that it is simply related to the overlap matrix (1)

$$
\Delta_{m \alpha J, m^{\prime} \alpha^{\prime} J^{\prime}}=\delta_{m m^{\prime}} \delta_{\alpha \alpha^{\prime}} \delta_{J J^{\prime}}+\sum_{p} M_{p}^{*}\left(m^{\prime} \alpha^{\prime} J^{\prime}, m \alpha J\right) .
$$

From (9) and (11) the expressions of $A$ and $\Delta$ look very similar; this allows them to be calculated in only one step in a numerical treatment. Once the matrices (1) and (5) are known one readily proceeds to calculate and diagonalize the matrix (3) to obtain the wavefunction (6).

Finally, with these wavefunctions one evaluates the desired transition matrix elements. In particular, the spectroscopic amplitudes for one-particle transfer reactions to states in the three-particle system is simply calculated from eq. (6) to get

$$
\left\langle\psi_{\beta} \mid \varphi_{m \alpha J}\right\rangle=\sum_{m^{\prime} \alpha^{\prime} J^{\prime}} X_{\beta}\left(m^{\prime} \alpha^{\prime} J^{\prime}\right) \Delta_{m \alpha J, m^{\prime} \alpha^{\prime} J^{\prime}} .
$$

We applied our method to the case of three identical particle system region, where shell-model calculation are available [7].

As far as an exact calculation is performed $\left(n^{\prime}=n\right)$ we are in complete agreement with the results of Mc Grory et al. [7] for both energies and spectroscopic factors.

In order to analyse the effect of drastic truncation we evaluated the overlap between the exact shell model wavefunction $\left|\psi_{\beta}\right\rangle$ and a restricted wavefunction $\left|\psi_{\beta}\left(N^{\prime}\right)\right\rangle\left(N^{\prime}\right.$ such that $\left.n^{\prime}<n\right)$, i.e.

$$
R_{\beta}\left(N^{\prime}\right)=\left\langle\psi_{\beta} \mid \psi_{\beta}\left(N^{\prime}\right)\right\rangle=\sum_{i=1}^{N^{\prime}} X_{\beta}^{\left(N^{\prime}\right)}(i)\left\langle\psi_{\beta} \mid \varphi_{i}\right\rangle
$$

which is easily calculated through (7) and (12).

The overlaps (13) depend both upon the number $N^{\prime}$ of states chosen in the basis and upon which states are chosen.

We found that a good choice is to take those few states with first order perturbation energy

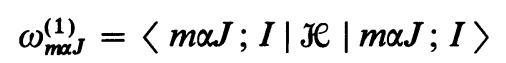


Table I. - Overlaps between the exact shell-model wavefunctions and those obtained in a truncated subspace for the first six $9 / 2^{-}$excited states in ${ }^{211}$ At. The two-body interaction is the second approximation of Kuo and Herling [9]. The dimension of the shell-model space is $n=80$. The total CDC 6600 computer time used to get these six shell-model wavefunctions was only 12 seconds for the exact case.

$\begin{array}{ccccccc}R_{\beta}\left(N^{\prime}\right) & \left|9 / 2_{1}^{-}\right\rangle & \left|9 / 2_{2}^{-}\right\rangle & \left|9 / 2_{3}^{-}\right\rangle & \left|9 / 2_{-}^{-}\right\rangle & |9 / 2-\rangle & |9 / 2-\rangle \\ - & - & - & - & - & - & - \\ R_{\beta}(15) & 0.9997 & 0.9992 & 0.9986 & 0.9846 & 0.9879 & 0.9981 \\ R_{\beta}(25) & 0.9999 & 0.9997 & 0.9996 & 0.9958 & 0.9963 & 0.9995\end{array}$

close to the energy value of interest. This is the essence of the weak coupling model [2].

For the low energy region of the spectrum, however, the states $P_{\alpha}^{+}(J)|0\rangle$ with $J=0^{+}, 2^{+}, 4^{+}, 6^{+}$and $8^{+}$ play a major role. In the table I we present the values of $R_{\beta}\left(N^{\prime}\right)$ for the first six $9 / 2^{-}$excited states in ${ }^{211} \mathrm{At}$ and for two values of $N^{\prime}$. The basis consists of the first $N^{\prime}$ states (8) chosen as above. As seen in this table, a relatively small number of basis states is enough to obtain a good description of the physical states (in this case $N=242$ and $n=80$ ). The influence of the collectivity of the two-particle states is seen especially in the ground state $9 / 2^{-}$. Taking only the basis state $\left|\varphi_{1}\right\rangle=\left|\mathrm{h}_{9 / 2} \otimes \mathrm{O}_{\mathrm{gs}}^{+}\left({ }^{210} \mathrm{Po}\right)\right\rangle$ we found

$$
R_{\mathrm{gs}}(1)=0.9981
$$

In conclusion, as far as the three identical particle system is concerned, the method presented in this letter provides a rapid calculation of the exact shellmodel solutions and to perform drastic truncations without loosing much precision in the description of the physical states.

More detailed analysis of the previous method and exact shell-model calculations with the full KuoHerling effective interaction [9] will be presented in ref. [10] for the ${ }^{211} \mathrm{~Pb}$ nucleus and in ref. [11] for the ${ }^{205} \mathrm{Hg},{ }^{205} \mathrm{Tl},{ }^{211} \mathrm{Bi},{ }^{211} \mathrm{Po}$ nuclei.

The equations we obtained giving the matrices $A$ and $\Delta$ for other systems, seem to show that our method is more efficient as compared to the standard shellmodel [1] the smaller the number of valence particles or the larger the number of active orbits.

(see also ref. [8]) .

\section{References}

[1] Brussaard, P. J. and Glaudemans, P. W. M., Shell model applications in nuclear spectroscopy (North-Holland, NewYork) 1977, p. 376.

[2] Ko, C. M., Kuo, T. T. S. and Mc Grory, J. B., Phys. Rev. $\mathrm{C} 8$ (1973) 2379.

ZUKER, A., International Conference on a Nuclear Structure and Spectroscopy (Amsterdam) 1971, p. 115

[3] Ring, P. and Schuck, P., Z. Phys. 269 (1974) 323;

SCHUCK, P., Z. Phys. 1279 (1976) 31.

[4] Bortignon, P. F., Broglia, R. A., Bes, D. R. and Liotta, R. J., Phys. Rep. 30C (1977) 305

[5] True, W. W. and MA, C. W., Phys. Rev. C 9 (1974) 2275.
[6] Liotta, R. J., Pomar, C. and Silvestre-Brac, B., Symposium on pure and applied Nuclear Physics (Stockholm) 1978. Contributed papers.

[7] Mc Grory, J. P. and Kuo, T. T. S., Nucl. Phys. A 247 (1975) 283.

[8] Arvieu, R., Bohigas, O. and Quesne, C., Nucl. Phys. A 143 (1970) 577.

[9] Kuo, T. T. S. and HeRLing, G. H., Naval Research Laboratory Report 2258 (Washington D.C.) 1971.

[10] Boisson, J. P., Silvestre-Brac, B. and Liotta, J. R., in preparation.

[11] Silvestre-Brac, B. and Boisson, J. P., in preparation. 